\title{
Norois
}

Environnement, aménagement, société

\section{Les outils graphiques paysagers, révélateurs des enjeux agricoles, dans un Plan Local d'Urbanisme}

Landscape tools to reveal agricultural stakes into an Urban Local Planning

\section{Claire Planchat-Héry}

\section{(2) OpenEdition}

1 Journals

\section{Édition électronique}

URL : https://journals.openedition.org/norois/2679

DOI : $10.4000 /$ norois. 2679

ISBN : 978-2-7535-1557-4

ISSN : $1760-8546$

\section{Éditeur}

Presses universitaires de Rennes

Édition imprimée

Date de publication : 15 décembre 2008

Pagination : 109-125

ISBN : 978-2-7535-0805-7

ISSN : 0029-182X

\section{Référence électronique}

Claire Planchat-Héry, « Les outils graphiques paysagers, révélateurs des enjeux agricoles, dans un Plan Local d'Urbanisme », Norois [En ligne], 209 | 2008/4, mis en ligne le 01 mars 2011, consulté le 14 janvier 2022. URL : http://journals.openedition.org/norois/2679; DOI : https://doi.org/10.4000/norois. 2679

(c) Tous droits réservés 


\title{
LES OUTILS GRAPHIQUES PAYSAGERS, RÉVÉLATEURS DES ENJEUX AGRICOLES, Dans un Plan Local d’Urbanisme
}

\author{
Claire Planchat-Héry \\ UMR MÉTAFORT, CERAMAC \\ (ENITAC, Université Blaise-Pascal), \\ Site de Marmilhat - 63270 LEMPDES \\ metafort.planchat@gmail.com
}

\section{RÉSUMÉ}

Quels sont les projets des agriculteurs et les enjeux d'une agriculture périurbaine à prendre en compte dans le futur zonage du Plan Local d'Urbanisme? Pour répondre à cette question posée par les élus de la Commune de Billom (Auvergne, France), nous proposons un dispositif participatif articulant divers outils graphiques de représentations des paysages comme supports de discussion entre exploitants agricoles et maîtres d'ouvrage du PLU. Notre hypothèse est qu'utiliser les représentations paysagères dans leur double dimension matérielle et idéelle, permet de faire dialoguer les acteurs sur les usages et les images de l'espace qui les supportent. Ce dialogue favorise la discussion des intentions des acteurs d'agir sur l'espace agricole, en prenant en compte les dynamiques du territoire aux différentes échelles.

MOTS CLÉ : représentations paysagères - démarche participative - projet d'aménagement - échelles d'intentions

\footnotetext{
ABSTRACT

Landscape tools to reveal agricultural stakes into an Urban Local Planning

How should a local plan for urbanisation take into account the projects of periurban farmers and the issuse they have at stake? To answer this question asked by the elected stakeholders of Billom (Awvergne, France), we propose to experiment participative approaches using various graphic designs of the landscapes as supports of discussion between farms owners and stakeholders of the Urban Local Planning. Our hypothesis is that using the landscape representations with their two folded nature of material and ideal objects might help stakeholders to talk together and discuss the uses and the images of their daily space. This dialog allows the various actors to better their common understanding, to act on agricultural space and to visualise, on various scales, territorial dynamics.
}

KEY WORDS : landscape representations - participative approaches - planning project - intentions scales 
La loi sur la Solidarité et le Renouvellement Urbain du 13 décembre 2000 a institué les Plans Locaux d'Urbanisme (PLU) en remplacement des Plans d'Occupation des Sols (POS). Un PLU ne doit plus seulement être une procédure planificatrice et normative produisant un zonage réglementaire pour la gestion d'un territoire, il s'agit d'un projet de territoire qui doit assurer, selon l'article L121-1 du code de l'urbanisme, «l'équilibre entre le renouvellement urbain, un développement urbain maîtrisé, le développement de l'espace rural, la préservation des espaces agricoles et forestiers, la protection des espaces naturels et des paysages ». Les modalités d'action pour maîtriser cet équilibre apparaissent dans un rapport de présentation associé à un Programme d'Aménagement et de Développement Durable (PADD). Ces orientations d'aménagement sont accompagnées de documents graphiques représentant la délimitation de zones et des règles qui y sont applicables. Pour les territoires périurbains, cet équilibre est difficile. L'espace agricole est souvent considéré pour sa valeur spatiale et foncière en tant qu'espace disponible, ce qui reste « en blanc » sur les cartes (Fleury, 2005).

Le territoire périurbain étudié ici, la commune de Billom en Auvergne, est caractérisé de « Tiers espaces », mi-ville, mi-campagne (Vanier 2003). Il est sous influence urbaine en termes socioéconomiques : tertiarisation des actifs, mouvements pendulaires vers l'agglomération, habitat pavillonnaire. Occupant plus de $50 \%$ de la surface, les espaces agricoles sont considérés comme un héritage d'une ruralité revendiquée à travers les discours des porteurs de projets d'aménagement (Planchat et Loudiyi, sous presse).

Bien que le législateur invite les décideurs à mettre en place des dispositifs de concertation pour la réalisation des documents d'urbanisme, les modalités de leur mise en œuvre demeurent très restrictives, d'autant plus pour des communes à faibles revenus fiscaux. En effet, le coût humain et technique d'une telle démarche demande des moyens auxquels ces communes ne peuvent pas toujours répondre. Il est difficile d'instaurer un dialogue spatialisé entre des intérêts privés et des intérêts collectifs, portés sur les espaces agricoles (Michelin, 2000) car cela nécessite d'appréhender des dimensions techniques (agronomiques, urbanistiques...), politiques voir psychologiques comme facteurs de réussite, pour une mise en action des intentions de gestion du territoire. Quels sont alors les nouveaux apports méthodologiques et leurs outils qui permettent de révéler ces diverses dimensions intégrées par les porteurs de projet pour argumenter leurs intérêts, leurs choix de gestion et d'aménagement?

Nous proposons d'utiliser les représentations du paysage dans le cadre de démarches participatives, pour partager ces dimensions afin de construire collectivement les éléments d'aménagement pour la gestion des espaces agricoles. En effet, nous supposons que l'usage de représentations paysagères facilite le dialogue pour la compréhension des intentions d'aménagement des porteurs du projet et pour la production des documents cartographiques réglementaires. Les outils graphiques paysagers, en tant que supports matériels et immatériels du territoire, permettent la construction et la combinaison d'objets spatiaux et sociaux utiles à la réflexion sur les dynamiques territoriales passées et futures. Nous considérons alors que les objets paysagers deviennent un « outil révélateur », dans le sens où leur considération permet de faire émerger des représentations spatiales et sociales d'un espace donné, par la production d'image et de sens, dépendantes de facteurs culturels et sociaux. L'observation directe de ces objets ou le contexte dans lequel ils sont intégrés revêt ainsi, une forte charge intentionnelle où la vision et la perception s'effectuent selon un dessein, un projet pour discerner, comprendre et agir. Notre objectif est de saisir cette charge intentionnelle à partir d'objets paysagers afin de mieux comprendre le projet des acteurs à intégrer dans le PLU.

Nous développerons le processus participatif que nous avons mis en œuvre au cœur du PLU de la commune de Billom. Nous avons intégré, durant cette procédure, une démarche pour aider les élus municipaux à prendre en compte les projets agricoles dans leur projet. À partir des débats menés entre porteurs du projet, élus et exploitants agricoles, nous discuterons les résultats obtenus, à travers une grille d'analyse des intentions de gestion du territoire à différentes échelles d’observation paysagère. 


\section{La question agricole dans le PLU du Billom, commune périurbaine de l'agglomération de Clermont-Ferrand}

La mise en œuvre d'un PLU nécessite de penser l'espace pour sa valorisation et son développement. Or sa construction relève de plusieurs difficultés auxquelles l'urbaniste et les décideurs doivent se confronter.

\section{LE CONTEXTE : Quelle Place POUR Les ESPACES AGRICOles DANS LES PLU?}

Au niveau réglementaire, le PLU présente un zonage cartographique déterminé, à partir d'un diagnostic du territoire et la définition d'intentions de gestion des diverses zones inscrites au PADD. Quatre catégories de sectorisation spatiale sont à définir par les porteurs du projet : les zones urbaines $[\mathrm{U}]$, les zones à urbaniser $[\mathrm{AU}]$ les zones naturelles et forestières $[\mathrm{N}]$ et les zones agricoles $[\mathrm{A}]$. Cependant, nous constatons qu'actuellement ces zonages réglementaires sont davantage réalisés comme un patchwork que comme un puzzle. Ils apparaissent davantage comme un découpage sectoriel des occupations de l'espace que comme une image cohérente et transversale d'un projet territoire intégrant une multiplicité d'espaces.

La lecture de divers rapports de PLU montre la difficulté des urbanistes à définir la question agricole, sa multifonctionnalité et ses divers acteurs à une échelle plus fine que celle du canton. En effet, pour des obligations de confidentialité, le recensement agricole ne permet pas d'obtenir des informations pour une commune de moins de 5 exploitants. De plus, on observe un amalgame entre les enjeux économiques et les enjeux spatiaux, négligeant ainsi de considérer les divers systèmes de production et leurs dynamiques. En effet, dans les rapports de présentation, l'agriculture est généralement évoquée dans le chapitre du développement économique en termes d'emploi et dans celui du diagnostic environnemental pour son rôle paysager. Les espaces agricoles sont caractérisés comme espaces non bâtis dont la seule définition d'intention reconnue dans l'article R 123 - 7 du code de l'urbanisme est de les protéger « en raison du potentiel agronomique, biologique ou économique ». Si on souhaite intégrer la question écologique et paysagère des activités agricoles, le zonage en $\mathrm{N}$ est alors recommandé. Il se présente ainsi la difficulté pour les porteurs du projet d'aménagement de réaliser une analyse précise et adaptée aux systèmes agricoles présents sur leur territoire, devant articuler des enjeux écologiques, paysagers avec des enjeux économiques strictement liés à la production.

\section{LA ZONE D’ÉTUDE : UNE COMMUNE PÉRIURBAINE À L'AGRICULTURE DIVERSIFIÉE}

La commune de Billom (4 575 habitants en 2007) appartient à la périphérie des $20 \mathrm{~km}$ au sudest de l'agglomération de Clermont-Ferrand (140700 habitants) (fig. 1). Il s'agit d'un territoire de 1696 hectares constitué à $66 \%$ par des espaces agricoles ponctuellement boisés (photo 1). Situé sur les terres noires de la plaine alluvionnaire de la Limagne, il est ponctuée de divers necks volcaniques lui offrant, au dire des élus communaux, « un cadre de vie de qualité ». Cependant sa proximité de Clermont, lui confère un caractère périurbain : un solde migratoire positif d'environ $2 \%$ entre 1999 et 2007, plus de $40 \%$ de la population active travaillant sur l'agglomération, environ 635 nouvelles constructions entre 1975 et 2003 dont $85 \%$ de type individuel ${ }^{1}$ et ayant augmenté de plus d'un quart la surface du bourg central ${ }^{2}$. Parallèlement, les différents secteurs agricoles font l'objet de multiples pratiques à différentes échelles et acteurs. Face à ces évolutions, la crainte de la destruction de cet espace agricole, de la perte de la qualité paysagère, est un thème récurrent des réunions des porteurs d'enjeux du territoire de Billom. Pour essayer d'intégrer ces éléments lors de la révision de leur POS en PLU, sur la période 2005-2008, les conseillers

1. Sources : Direction Régionale de l'Équipement.

2. Analyse diachronique des photos aériennes 1999 et 2004 - IGN BD Ortho. 

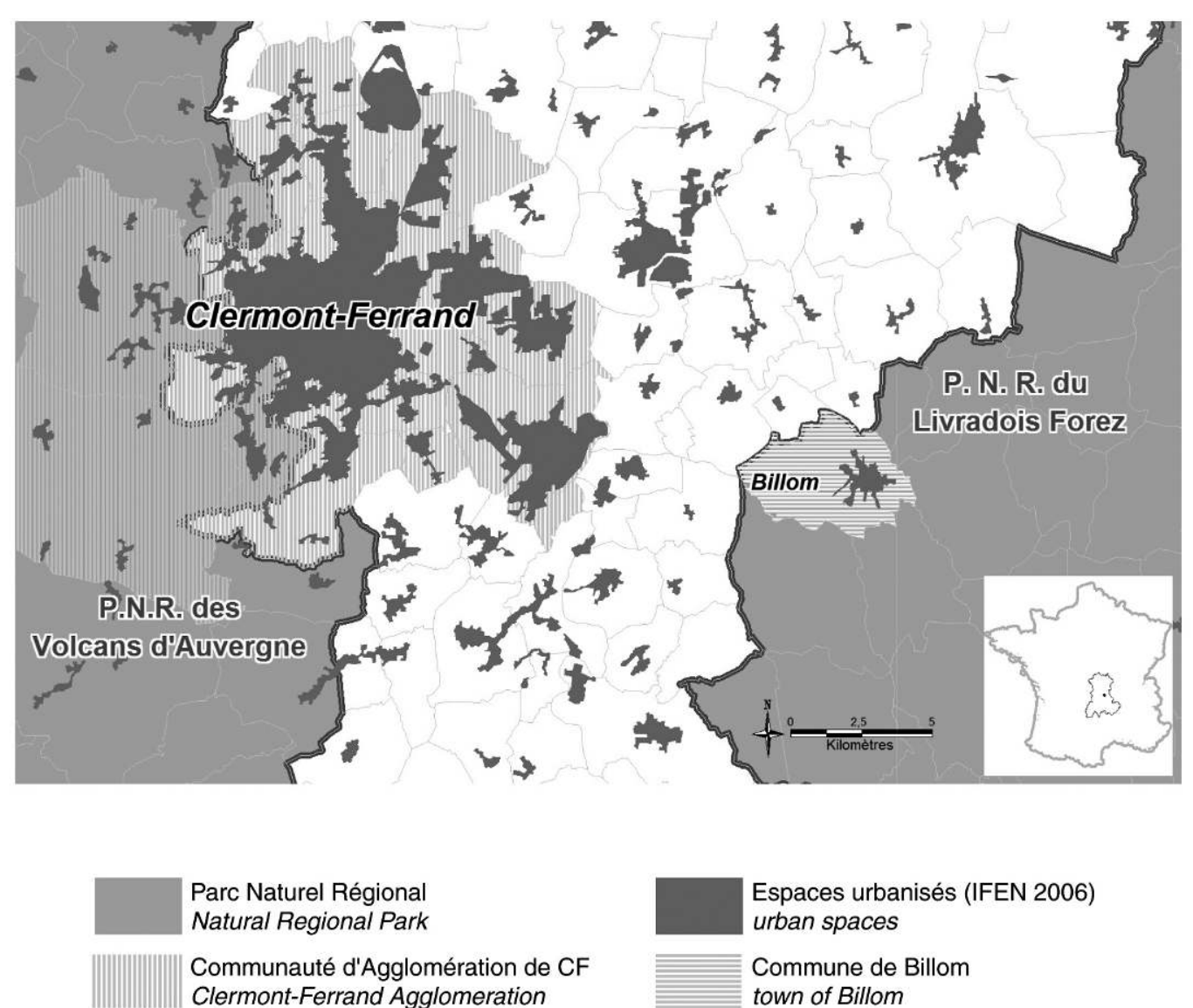

Parc Naturel Régional
Natural Regional Park

Communauté d'Agglomération de CF Clermont-Ferrand Agglomeration

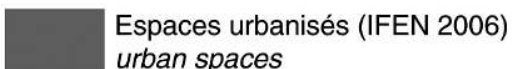

urban spaces

Commune de Billom town of Billom

Figure 1 : Carte de localisation de la commune de Billom Location map

municipaux, soutenus par la Chambre d'Agriculture du Puy-de-Dôme, ont accepté que nous leur proposions un Itinéraire Méthodologique de Vision Prospective (IMVP) des espaces agricoles de leur commune, devant répondre aux objectifs suivants : connaître et faire connaître l'activité agricole et ses espaces d'action; par le biais de la participation, améliorer la qualité des décisions pour les élus et l'implication à la décision pour les agriculteurs; dégager des orientations collectives autour d'un projet de développement durable; intégrer notre dispositif dans les phases de concertation du PLU.

Les divers changements des orientations agricoles ont conduit ce territoire à de profondes mutations. Les remembrements réalisés entre 1975 et 1999 ont accentué les risques de déséquilibres fonciers importants entre urbanisation et grandes cultures. La commune de Billom a subit une forte diminution du nombre d'exploitations de moins de 50 ha ayant pour conséquence la création de groupements agricoles de plus de 150 ha répartis sur les communes limitrophes de Billom. La domination des grandes productions céréalières, notamment du blé, n'empêche pas d'autres productions $^{3}$ : céréales (73\% des productions), betterave à sucre $(9 \%)$, colza (4\%) et surfaces pâturées $(5 \%)$. La vigne, en forte diminution, résiste sur certaines pentes des buttes volcaniques environnantes, à la fois pour une production privée et labellisée. La part laissée à la production

3. Sources : enquêtes menées auprès de 16 agriculteurs propriétaires de parcelles sur Billom en 2006. 
animale n'est pas à négliger, $1 / 4$ des agriculteurs sont concernés. Les cheptels bovins laitiers sont un peu plus présents que les cheptels ovins. Cette diversité crée un potentiel local pour la vente directe, fédérée par la présence d'associations locales.

La commune de Billom bénéficie de 21 exploitants en 2000, contre 69 en 1979². En 1999 le regroupement est privilégié, réduisant fortement l'installation individuelle. L'âge croissant des petits exploitants (48\% ont plus de 60 ans) a reconfiguré la répartition parcellaire. $49 \%$ de ceux qui cessent leur activité laissent leurs terres aux exploitants déjà présents, $18 \%$ associent leur famille, enfants ou conjoints. $31 \%$ n'ont pas de successeur pour leur exploitation.

À partir d'un diagnostic préalable que nous avons effectué, ce territoire présente trois typologies de configurations agricoles et paysagères, selon divers enjeux (photo 1) :

- le hameau de Tinlhat et ses environs : sur ce secteur, l’agriculture céréalière domine. Les drains et leurs ripisylves, ainsi que le hameau groupé et quelques domaines agricoles dispersés structurent le paysage. Il est intéressant de voir comment l'urbanisation du hameau s'est peu développée en comparaison au centre bourg. L'ancien POS a fortement contrôlé la pression foncière liée à l'avancée du front de l'agglomération clermontoise. Le village est aujourd'hui un îlot de la plaine remembrée dont le développement dépend notamment des intérêts de nombreux exploitants à la retraite, désireux de céder leurs terrains en espace constructible.

- Le secteur est présente une mosaïque paysagère avec une urbanisation dominante. Le centre bourg de Billom ne connaît pas de profondes modifications tandis que sa périphérie sud s'agrandit. Le nord du bourg est plus soumis à un enjeu de développement de zones artisanales. Au sud-est il présente un équilibre fragile entre des espaces de polyculture élevage, des espaces boisés et la proximité de cours d'eau créant des zones inondables. Le maintien des espaces arborés est un autre enjeu de ce secteur, notamment pour les ripisylves des principaux cours d'eau.

- Le secteur central est caractérisé par les necks volcaniques appelés Turlurons. Cet espace est ponctué de zones protégées : ZNIEFF, NATURA 2000 et boisements classés. Sur les pentes, on observe deux types d'agriculture, d'une part du maraîchage avec vignoble, verger et ail, et d'autre part, une agriculture intensive céréalière. Les bas de pentes s'urbanisent, malgré de fréquents écoulements de boues liés aux forts ruissellements que les parcelles cultivées remembrées ne retiennent plus.

Photo 1: Bourg central et lotissement de Billom Central burg and new private housing of the town of Billom

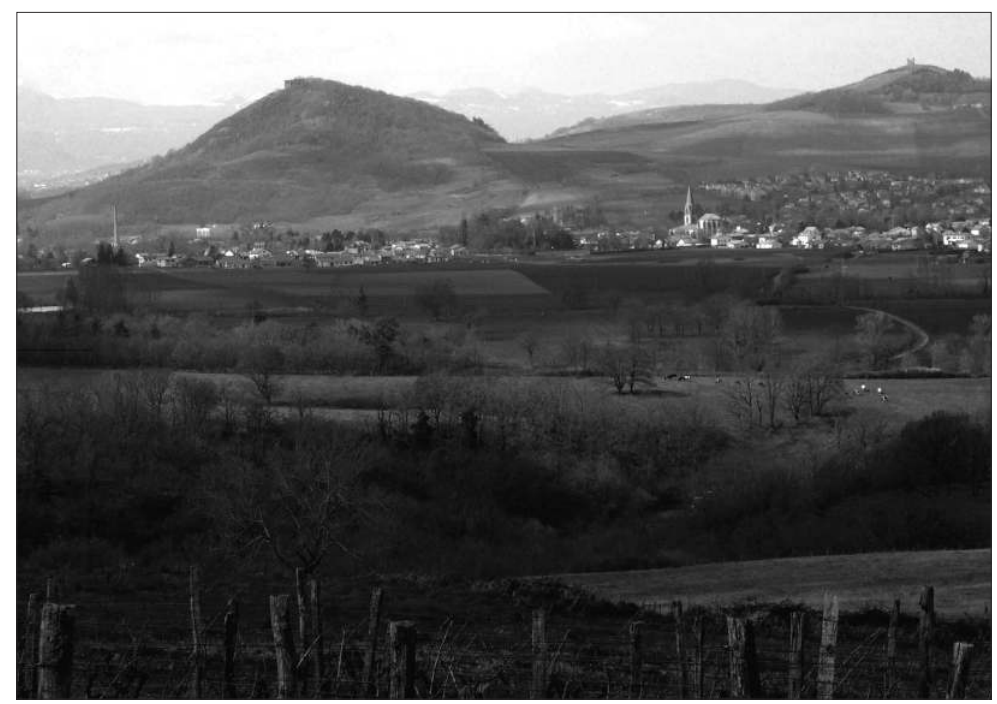

4. Recensement Général de l'Agriculture 2000. 
Pour considérer au mieux la question agricole sur l'ensemble du territoire, nous avons proposé de fédérer les acteurs autour d'outils participatifs et de dialogue au cœur de la procédure du PLU. Pour concilier participation des individus et caractère prospectif des actions d'aménagement, nous avons construit un protocole itératif nommé Itinéraire Méthodologique de Vision Prospective.

La méthode : l'Itinéraire Méthodologique de Vision Prospective

L'IMVP permet l'articulation de différentes méthodes et outils de traitement de l'information, mais aussi de «mobilisation des acteurs d'un territoire autour de la production de représentations spatiales, pour construire progressivement une vision partagée et stratégique du territoire » (Lardon et al., 2001).

Le protocole de Vision Prospective que nous avons proposé consiste, lors de moments clés de la procédure, à construire et à articuler des outils de représentations spatiales et paysagères. L'implication du chercheur s'inscrit à deux niveaux. Le premier correspond à l'animation pour fédérer les acteurs dans la démarche. Le second concerne l'observation et le décryptage des représentations et intentions sur les objets retenus par les acteurs lors de l'expérimentation et ceux intégrés dans la procédure. L'intérêt de l'IMVP est donc de s’inscrire dans un processus d'aide à la décision (Joliveau, 2001) en amont des phases clés d'un projet de territoire.

L'IMVP est constitué de 5 étapes dont les étapes participatives peuvent être renouvelées à différentes phases de la procédure et avec différents acteurs :

Étape 1 - Diagnostic de territoire et paysager réalisé par le chercheur. Il s'agit de recueillir les premiers enjeux de l'agriculture à partir de lecture de paysage sur le terrain, de cartes, d'iconographies issues d'études, de publicités et de rencontres. On aboutit à la construction d'un premier catalogue d'objets paysagers (Planchat et Loudiyi, sous presse) à partir duquel on réalise une synthèse graphique en cartographies, maquette en carton $3 \mathrm{D}$ et blocs diagrammes paysagers (Michelin, 2000). Ces supports sont mobilisés pour les phases participatives individuelles et collectives de notre démarche.

Étape 2 - Enquêtes par questionnaires et entretiens individuels semi-directifs. L'acteur déconstruit les éléments du paysage à travers son parcours, ses pratiques et ses projets. Le chercheur analyse cette production en termes de représentations spatiales et sociales.

Étape 3 - Atelier participatif 1, la «vision prospective négative ». La déconstruction collective des perceptions du territoire, à partir de la question de «ce qu'on ne veut pas voir », permet d'argumenter et d'anticiper les évolutions à venir, liées à des actions individuelles et collectives. L'intérêt de l'approche par la négative est le caractère indirect de cette vision qui ne se définit que par rapport à son contraire. Elle permet de plus facilement révéler les éléments qui ne sont pas fondés sur l'observation immédiate du territoire, mais sur la connaissance et les intentions que l'on en a à partir des paysages perçus et vécus par les acteurs locaux. L'illustration graphique principalement utilisée est le bloc-diagramme paysager Trois raisons orientent notre choix d'utiliser le bloc diagramme paysager comme support de dialogue des enjeux territoriaux. Tout d'abord il s'agit d'une représentation intermédiaire entre une vue 'de dessus', cartographique, et une vue 'de dedans', à la tangente du regard. Ce mode de visualisation est facilement lu par la majorité des acteurs. De plus il peut être utilisé comme outil complémentaire entre une carte et une photo. Il permet de représenter à la fois les espaces non visibles d'une photo, la volumétrie, des entités paysagères, mais aussi peut être le support de représentations de flux et de dynamiques. Ensuite, le bloc diagramme permet la représentation des éléments paysagers selon une certaine abstraction de la réalité, mieux expliquée par les objets paysagers, que peut permettre un dessin, mais aussi une maquette car chaque élément est construit individuellement. Enfin, c'est un outil facilement utilisable en réunion ou sur le terrain ce qui lui permet ainsi d'être manipulé dans un cadre participatif comme support de lecture et de positionnement des objets paysagers porteurs d'enjeux pour le développement du territoire. Le chercheur en tant qu'animateur aide les acteurs à dessiner sur le bloc les objets paysagers qu'ils ne veulent pas voir. L'apport des éléments de chacun après débat, offre un modèle de territoire que l'on ne souhaite pas, construit à partir d'une vision partagée. 
Étape 4 - Atelier participatif 2, la « vision prospective positive » ou « ce qu'on veut voir ». Nous proposons aux acteurs de mobiliser leurs représentations pour la description et l'analyse des processus de différenciation qui s'opèrent sur leur commune et d'envisager l'identification d'enjeux territoriaux issus des connaissances, des pratiques et des projets individuels et collectifs. La conversion du négatif en positif amène les acteurs à hiérarchiser, sur une carte vierge, leurs intentions mais aussi à les positionner spatialement. En effet, chaque élément en atelier 1 peut être utilisé en atelier 2 comme objet décrivant un espace et des pratiques, mais aussi comme élément autonome de toute activité selon s'il est considéré comme appartenant ou non au territoire.

Étape 5 - Ce que les décideurs ont retenu pour la procédure. Les représentations graphiques et des éléments de dialogue produit lors des ateliers participatifs sont analysés selon leur intégration ou non dans les documents du projet.

Notre démarche a été mise en œuvre en parallèle de la démarche du PLU, sur deux ans (20062007). Itératif, l'IMVP a pu être appliqué sur deux phases d'intervention tout d'abord avec les agriculteurs et les élus puis avec le conseil municipal (tableau 1), équivalant au total à une période de trois mois.

\begin{tabular}{|c|c|c|c|c|}
\hline & \multicolumn{2}{|c|}{2006} & \multicolumn{2}{|c|}{2007} \\
\hline & ENQUÊTE & ATELIER & ENQUÊTE & Atelier \\
\hline Agriculteurs & 21 & $\begin{array}{c}16 \text { (dont } 5 \text { retrai- } \\
\text { tés) }\end{array}$ & & 1 (élu) \\
\hline Élus & & $\begin{array}{l}2 \text { (maire et } 1^{\mathrm{er}} \text { ad- } \\
\text { joint })\end{array}$ & 14 & 1 \\
\hline $\begin{array}{l}\text { conseillers et } \\
\text { adjoints munici- } \\
\text { paux }\end{array}$ & & 2 & & 8 \\
\hline $\begin{array}{c}\text { Organismes décen- } \\
\text { tralisés }\end{array}$ & & $\begin{array}{c}\text { (2 Chambre. } \\
\text { d'agri., } 1 \text { DDE) }\end{array}$ & & \\
\hline $\begin{array}{l}\text { Collectivités terri- } \\
\text { toriales }\end{array}$ & & $\begin{array}{c}2 \text { PNR, } 1 \text { commu- } \\
\text { nauté de commu- } \\
\text { nes }\end{array}$ & $\begin{array}{l}1 \text { communauté de } \\
\text { communes }\end{array}$ & $\begin{array}{l}1 \text { communauté de } \\
\text { communes }\end{array}$ \\
\hline Chercheur & & 2 & & 2 \\
\hline $\begin{array}{l}\text { Associations envi- } \\
\text { ronnement et patri- } \\
\text { moniales }\end{array}$ & & 2 & & 1 (élu) \\
\hline $\begin{array}{l}2 \text { Architectes/ urba- } \\
\text { nistes }\end{array}$ & & 2 & 1 & 1 \\
\hline TOTAL & 21 & 29 & 16 & 15 \\
\hline
\end{tabular}

Tableau 1 : Effectifs des ateliers participatifs Number of participants at the workshops

En 2006, la première phase s'est déroulée en amont de la rédaction du diagnostic et du PADD. Un premier atelier participatif a été réalisé à partir de divers supports graphiques proposés et produits (figure $2 \mathrm{a}$ et $\mathrm{b}$ ).

Suite à l'intérêt porté à cette action participative par certains élus de Billom et dans un objectif d'aide à la décision sur le zonage final du PLU, une seconde démarche a été commandée en 2007 uniquement pour les élus du conseil municipal. Les étapes 2 et 4 de l'IMVP ont pu être de nouveau pratiquées jusqu'au traçage collectif de prototypes du zonage finaul du PLU (figure 2c et d). 


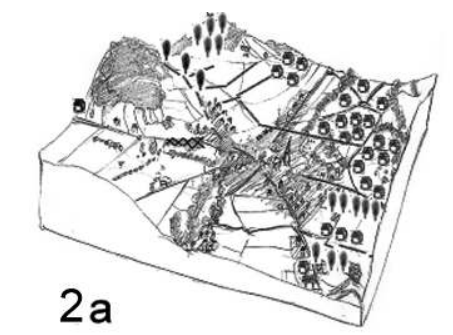

Ils ne voulaient pas voir - they don't want to see:

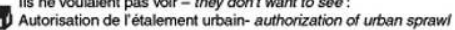

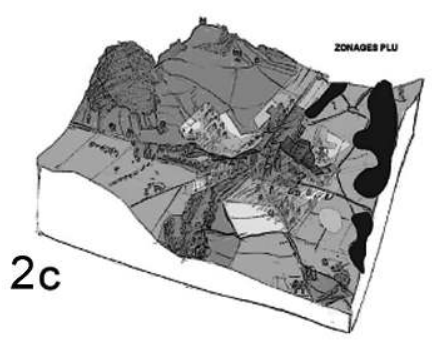

(1) Aucune action pour les bâtiments en ruine ou très anciens - No project for downfall and old buildings

$\times \subset \times$ Coupe abusive de peupliers sans replanter - abusive cut of poplars without replanting

Plantations d'arbres sur les crêtes - plantations of trees on the peaks

Alignements de noyers - alignments of walnut trees

- Création de nouveaux sentiers- new paths
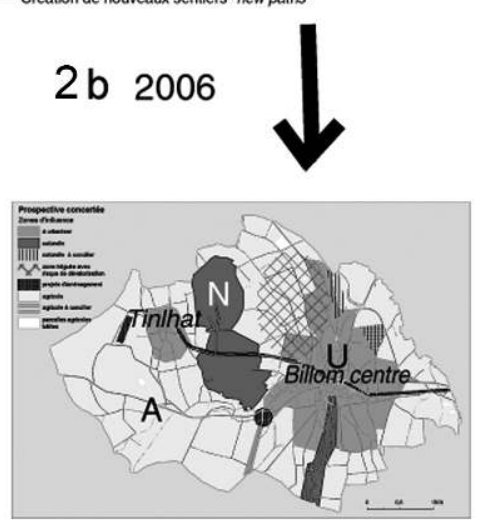
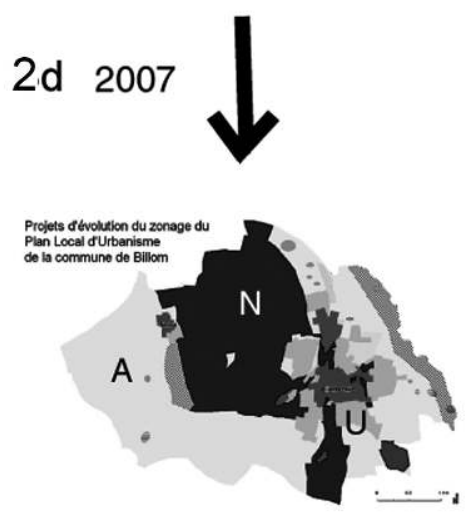

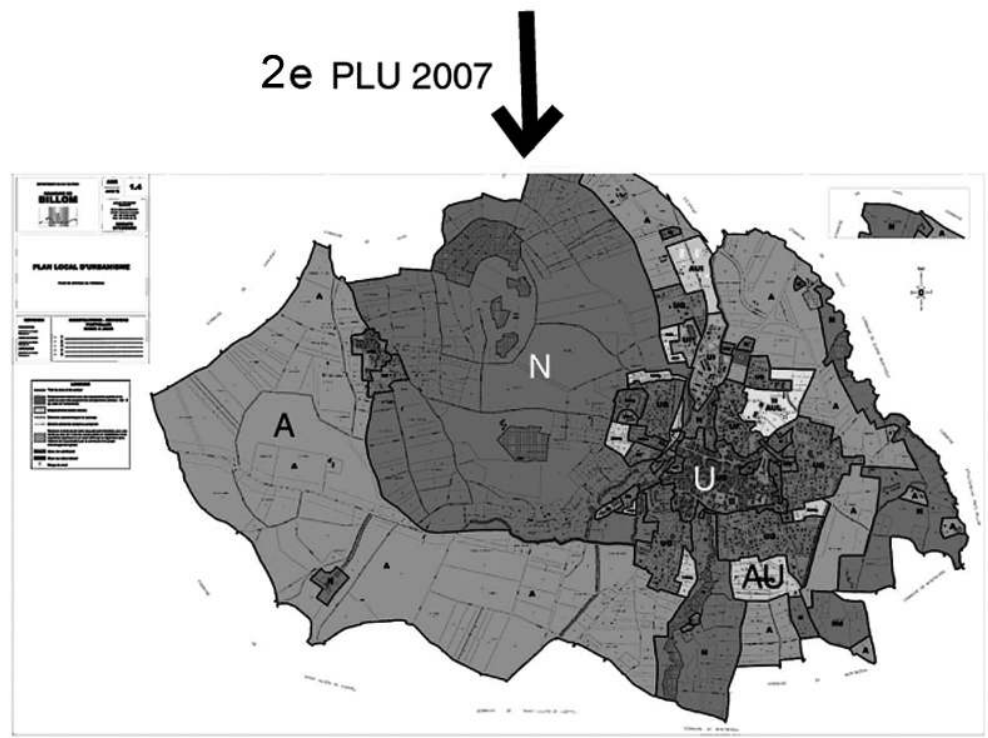

Figure 2 : Blocs diagrammes paysagers soumis à discussion pour une prospective à 5/10 ans et cartographies produites

3D landscape patterns subjected to discussion for a scenario into 5/10 years and maps drown. 
Le suivi de la procédure, depuis la constitution du rapport de présentation jusqu'à l'enquête publique, a permis d'analyser les représentations obtenues et leur intégration au projet de territoire(carte finale du PLU, fig. 2e). Nous pouvons ainsi étudier l'expression des types d'orientations qui résultent de certaines actions individuelles et collectives, mais aussi mesurer les éléments qui ont fait et qui font territoire agricole aujourd'hui pour ces acteurs.

La participation des habitants n'a pu être directement intégrée à l'expérimentation pour des raisons d'échantillonnage et de représentativité, mais aussi pour répondre à la demande de la Chambre d'Agriculture et du maire d'interroger en priorité les agriculteurs. En revanche, le regard protestataire de la population sur le PLU a pu être pris en compte lors de l'enquête publique réalisée en 2008, en fin de procédure.

Nous ne pouvons présenter l'ensemble des productions obtenues grâce à cette démarche. Nous retiendrons les résultats obtenus aux étapes 3 à 5 de l'IMVP, à savoir les intentions d'actions et leurs échelles d'observation débattues. Nous apportons ainsi un éclairage sur certains objets des paysages de Billom qui ont permis de faire émerger les choix des types de zonage des espaces agricoles enchâssés entre logiques de périurbanisation, de préservation paysagère et de production agricole.

\section{Les différentes échelles de la représentation de l'agriculture dans un PLU}

Les représentations graphiques utilisées lors des ateliers participatifs ont permis de mettre en dialogue les éléments et les évolutions possibles du territoire. Nous proposerons une lecture de ces résultats à travers notre grille d'analyse combinant échelles d'observation des objets paysagers (fig. 4) et les intentions d'actions sur ces objets (fig. 4). Nous analyserons également les enjeux des débats pour mieux évaluer ce qui a été retenu par les décideurs dans le PLU.

\section{DE L’ÉCHELLE DE L’obJET PAYSAGER À SA DIMENSION SYMBOLIQUe}

Parmi les trois échelles d'observation paysagère (aménagement, opération, objet), nous constatons (figure 4) que l'échelle de l'objet lui-même est la plus sollicitée. Elle s'applique principalement sur les intentions de mise en valeur des éléments identitaires et familiers : un résidu de parcelle

Figure 3 : Les échelles d'intention

Intention scales
Objet - Object : éléments individuels (maison, champ, arbre ...) ou groupement homogène (forêt, exploitation, ....)

Opération- Land Settlement : objets reconnus en tant qu'unité dans un but de requalification (place publique, jardin, ...)

Aménagement - Planning : imbrication de plusieurs unités géographiques, souvent cités par " grand paysage ".

Symbolique - Symbolic : liée par analogie à une signification abstraite, voire une métaphore ou une métonymie 
de vigne à maintenir pour une production familiale, une grange en centre bourg pouvant devenir habitat... Ces éléments ne sont plus seulement repérés comme patrimoine à préserver mais on observe, par l'analyse des intentions, la prise en compte par les acteurs de Billom de nouvelles attentes et changements sociaux qu'elles engendrent.

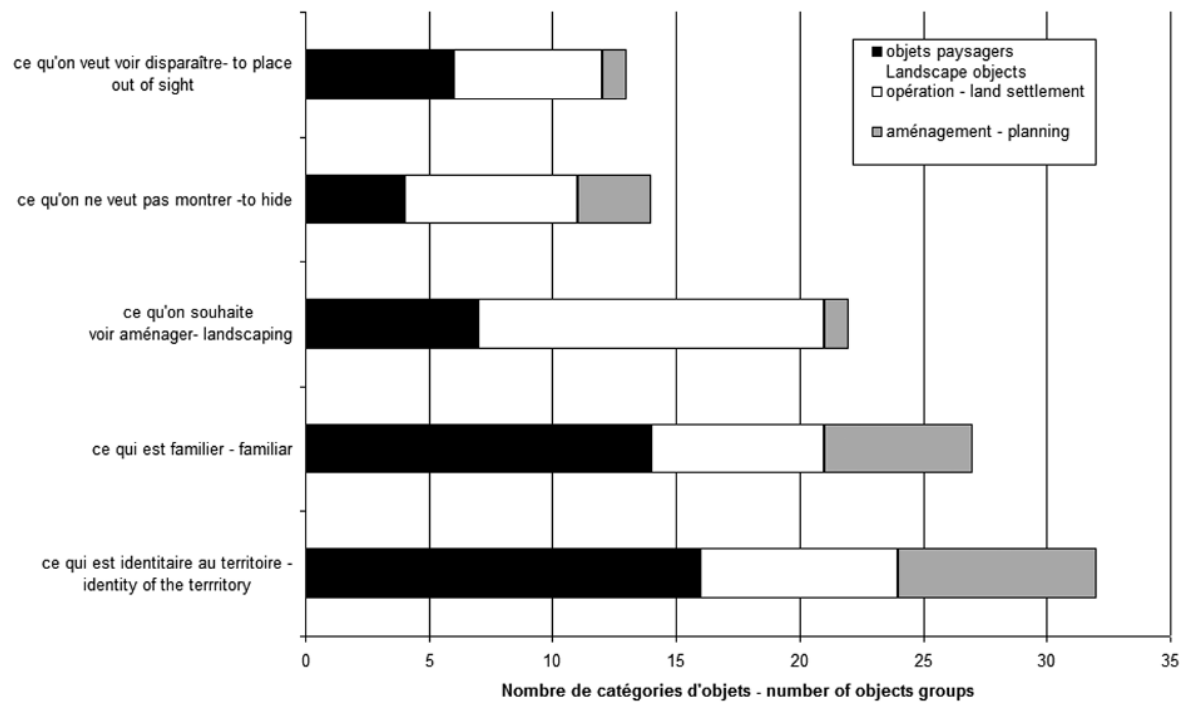

Figure 4 : Répartition des éléments paysagers par échelles d'intentions Distribution of the landscape elements by intentions scales

La description des éléments de l'échelle de l'objet permet de révéler leur rôle dans les mutations des structures paysagères, les enjeux dans lesquels ils sont impliqués, les stratégies individuelles face aux stratégies collectives vis-à-vis d'un même objet. L'intention de «faire disparaître » s'applique alors facilement à l'échelle de l'objet.

Un autre constat concerne l'utilisation de la dimension symbolique non pas d'un objet, mais des images qu'il évoque, en réorientant ainsi les intentions à porter à cet objet. Les débats et stratégies, territoriales et paysagères, mobilisées autour de l'objet « arbre » et de ses déclinaisons illustrent ce propos. À partir du bloc-diagramme paysager de l'atelier de 2006 (figure 2a), l'arbre a été représenté sous différentes catégories d'objets, soient de manière ponctuelle, linaire ou surfacique : alignements de noyers, peupleraies, petits massifs boisés.

L'arbre a tout d'abord été considéré comme structure forestière. Ainsi le boisement présent au sommet des Turlurons a été en premier débattu sur l'intention de coupes ou de plantations arborées. Depuis le dernier remembrement de 1999, le conflit autour des plantations subsiste. En effet, il était prévu de planter certaines parcelles pour favoriser la structure paysagère des Turlurons et surtout diminuer les écoulements d'eaux. Aujourd'hui, les exploitants de ce secteur sont d'accord pour ne pas planter sur les sommets.

Les élus ont alors profité de l'objet de ce débat pour révéler aux propriétaires agricoles, le projet communal de sentier de randonnée entre Tinlhat et Billom centre. Ce projet porte sur la mise en valeur des chemins existants par la plantation d'alignements de noyers. Ce choix est débattu, car pour certains agriculteurs, ces plantations gêneraient le passage des engins agricoles. Les élus se coalisent pour proposer l'idée de conserver, en l'état, le chemin entre les Turlurons avec quelques plantations. Nous constatons que pour ces derniers, l'idée de plantation de noyers s'associe à la symbolique de 
l'arbre en lien avec l'intention de révéler ce qui est familier. L'un d'entre eux cite à ce propos : «Il y a plein de paysage autour des champs, des cultures, des vignes autour des chemins... il y avait plein de noyers et plein de vigne, avec le remembrement, les noyers sont noyés! »

Le débat a également porté sur les plantations de peupliers qui ont récemment fait l'objet d'une coupe définitive le long de certaines voiries et de plantations sur quelques parcelles. Pour des exploitants, les peupleraies et les haies peuvent financièrement être intéressantes, en termes de primes à la plantation. Cependant, la majorité d'entre eux ne souhaite pas ce type d'espèce, qui demande beaucoup trop d'entretien, notamment à proximité des drains. Pour les élus intercommunaux, la silhouette des peupliers rappelle celle des cyprès de toscane au point de qualifier le territoire de Billom de Toscane Auvergnate (Sycomore, 2007).

Le groupe des élus souligne alors le rôle des agriculteurs en tant «qu'agents d'entretien du paysage » permettant de préserver l'ouverture des paysages de la plaine céréalière. Le maintien des espaces ouverts dépend certes de la production céréalière intensive, mais aussi des pratiques d'élevages par des exploitants retraités ou pluriactifs qui ne sont pas présentes dans le discours des élus.

La thématique arbre jouera un rôle primordial dans l'atelier de 2007 dans le choix des zonages entre $\mathrm{A}$ et $\mathrm{N}$ (figure 2c). En effet, les zones bocagères et maraîchères seront classées en $\mathrm{N}$ du fait des ambiances paysagères qui fait référence à la dimension symbolique. En revanche, les zones céréalières appartiendront à une zone $\mathrm{A}$, du fait de la visibilité de la production (Sycomore, 2007). Ces approches montrent la finesse des échelles d'appréciation des espaces, entre des rapports intimes, familiers ou productifs à l'espace.

L'intérêt de classer en zone $\mathrm{N}$ les Turlurons est perçu différemment selon les agriculteurs, les élus et l'urbaniste. Pour les premiers (figure 2b), les avis sont très partagés entre exploitants notamment sur le fait de «surprotéger » cette zone. Il semble pertinent pour la majorité de respecter au plus prêt l'intérêt de la terre agricole et de ne classer en $\mathrm{N}$ que les espaces classés et le site Natura 2000. À la seconde démarche participative, les élus choisissent de recouvrir l'ensemble des Turlurons en zone $\mathrm{N}$ au titre d'éléments paysagers remarquables, ce qui révèle (figure $2 \mathrm{~d}$ ) des intentions identitaires, au sens d'une reconnaissance collective d'éléments singuliers. L'urbaniste approfondit la question en élargissant ce dernier zonage pour concilier protection écologique des bas de pentes (figure 2e). Ces enjeux ne sont pas seulement liés à la production, mais bien à des pratiques individuelles ne pouvant toutes être réglementées dans un document d'urbanisme.

Les controverses entre élus et agriculteurs nous montrent l'intérêt de mobiliser les représentations graphiques permettant d'articuler les différentes échelles d'observation. En effet, mobiliser l'échelle de l'objet n'empêche pas le conflit et ne privilégie pas non plus le consensus. Cependant, le partage des connaissances et les échanges sur les diverses pratiques à partir d'un même objet (i.e. la haie) favorise un débat argumenté sur les intentions soumises à l'échelle d'opération et d'aménagement (i.e. l'ouverture du paysage).

\section{DE L’ÉCHELLE D’opÉRATION À L'ÉCHELLE D’AMÉNAGEMENT}

Le graphique des échelles d'intention (figure 5) indique bien que l'échelle d'opération est la plus pertinente pour révéler les intentions d'aménager pour le changement. Cette échelle apparaît davantage dans le discours des élus sur leurs intentions de revaloriser un ensemble d'objets paysagers composant une unité. Alors que l'échelle d'aménagement est plus mobilisée sur les intentions de préservation des éléments familiers et identitaires.

Nous constatons que l'articulation entre échelle d'opération et échelle d'aménagement indique surtout l'écart entre l'image du projet d'aménagement que vont argumenter les élus, et l'opérationnalité, soit la pratique de cet aménagement que vont plutôt justifier les agriculteurs. On observe alors des allers-retours entre l'échelle d'opération et celle d'aménagement, l'une permettant d'argumenter l'autre. L'échelle d'opération va permettre de décrire des projets répondant à la demande 
d'évolution du territoire justifiant la nécessité de l'inscrire à une vision élargie que représente l'échelle d'aménagement. Cette dernière renvoie à une échelle sociétale du paysage, multi-acteurs et multiforme dont l'espace agricole va davantage être réfléchi en tant qu'indicateur de ruralité. Du fait d'une observation élargie du territoire, cette ruralité est liée à des propos génériques sur la nature et des discours scientifiques et politiques (Jean et Dionne, 2007). L'exemple du débat sur les aménagements routiers pour le développement de la ville illustre ce propos.

En 2006, certains exploitants ont posé le problème de l'augmentation du trafic routier face au développement de l'agglomération clermontoise. Sur ce point, la discussion entre élus et agriculteurs a porté sur le fait que l'axe principal entre Billom et Clermont-Ferrand ne doive pas se développer davantage et rendre encore plus difficile la circulation des engins agricoles. Les élus ont alors profité du débat pour présenter leur projet de carrefour giratoire (figure 2b) en prévision des futurs trafics liés au développement de l'agglomération et de l'accueil sur la commune de nouveaux habitants. En dépit des craintes des agriculteurs, les élus en 2007 ont justifié les secteurs à urbaniser légitimant l'intérêt général. C'est précisément la question de "l'intérêt général ». qui soutient les choix des décideurs.

Déclinée par l'art. L-121-9 du code de l'urbanisme, le PLU doit en effet faire figurer tout projet d'intérêt public à savoir tout projet d'ouvrage, d'équipement, mais aussi de protection du patrimoine naturel ou culturel pour la valorisation et l'aménagement des ressources naturelles, agricoles et rurales. La législation illustre bien cette difficulté à penser pour le collectif entre échelle d'opération et d'aménagement : accepter d'imposer un projet consommant de l'espace agricole sur un secteur mais penser protection de l'agriculture au niveau global. Cela soulève les incompréhensions entre agriculteurs et élus que peut engendrer une intention de préservation des espaces au nom de la nature, du paysage, en opposition au développement agricole et économique. C'est particulièrement cet intérêt qui est promu pour définir les intentions à l'échelle d'aménagement.

Ainsi en 2007, la volonté des élus est de préserver le secteur de Tinlhat dans son état en conserver la silhouette du bourg sans extension (figure 2c). Pour l'urbaniste, il s'agit de « combler les dents creuses ». Il semble intéressant de remarquer que certains élus affirment que la «qualité de vie de Billom a su être préservée », que « les habitants ont pu s'intégrer », que Billom n'est pas encore une " cité dortoir comme les communes en deuxième couronne de Clermont-Ferrand », ayant connu une vague d'urbanisation pavillonnaire dans les années 1970. Cet héritage que l'ancien POS a permis n'est pas mis en avant, tandis que le nouveau PLU va au final peu modifier cet ancien zonage. Cependant, le patrimoine bâti actuel ne suffit pas à l'accueil de nouvelles populations en quête d'espace et d'habitat individuel, ce qui justifie l'intérêt pour les élus de développer les zones constructibles sur l'espace agricole du secteur sud du centre bourg, au titre de « l'intérêt général de la commune » (figure $2 \mathrm{~d}$ ). Les exploitants savent que le foncier agricole est déjà soumis à certaines pressions, et selon les projets personnels, peut facilement changer de vocation. Ils ne souhaitent donc pas voir se développer des îlots d'habitat disséminés dans le paysage notamment sur la partie nord-est composée de bonnes terres (figure 2a).

En conclusion de la démarche élaborée en 2007, cinq orientations pour la gestion des espaces agricoles ont été proposées :

- se prémunir de l'avenir en définissant des critères adaptés à d'éventuelles demandes d'installation de nouveaux agriculteurs en zone A;

- utiliser l'espace agricole pour contrôler le développement urbain (cas du hameau de Tinlhat)

- prévoir la reconversion des bâtiments agricoles isolés en habitat pour éviter sur certains secteurs leur délabrement;

- pourvoir étendre à l'avenir le coefficient de surface à bâtir à partir de l'existant ;

- mobiliser le zonage en $\mathrm{N}$ des espaces agricoles pour justifier l'intérêt général de la préservation des paysages. 
Si l'enjeu final des élus municipaux consistait à pouvoir prendre en compte les différentes représentations spatiales et sociales des espaces agricoles, ils ont surtout mobilisé les résultats de l'IMVP pour mieux construire leur argumentaire et essayer d'articuler à différentes échelles d'appréciation et de thématiques leur proposition de planification. La carte finale du PLU (figure 2e) traduirait les propositions d'avenir retenues devant appréhender les diverses possibilités d'évolution de l'agriculture de Billom. Or une lecture plus approfondie du document final, et plus particulièrement la répartition des zonages $\mathrm{A}$ et $\mathrm{N}$, soulève diverses questions sur la non-prise en compte d'enjeux agricoles qui auraient pu être importants pour les agriculteurs. Nous proposons ainsi une lecture critique du rapport et du zonage afin d'analyser quels objets et intentions issus de notre démarche ont été incorporés.

\section{Discussion}

Si le PLU peut inciter à la mise en place d'une agriculture durable en prenant en considération les projets de certains agriculteurs, la confrontation entre les élus et les agriculteurs à partir des représentations spatiales et sociales d'un même territoire, nous montre plusieurs difficultés. Tout d'abord, les actions sur l'espace agricole à différentes échelles, depuis la sphère individuelle à la sphère sociale, depuis la compréhension des systèmes agricoles et leurs acteurs, à la gestion communale ne sont pas intégrée dans le PLU. Ensuite, l'urbaniste maintient des représentations du rural à travers une image bucolique des éléments spatiaux. Par exemple, concernant les aménagements routiers on peut lire au cœur du PADD : «La route de M. présente une image plutôt agréable. Elle affiche une trame arborée, quelques prairies et une perception un peu "villageoise" de la ville. »C'est bien le contraste des arguments proposés pour un zonage en A ou en $\mathrm{N}$ qui peut être remis en question.

\section{QUELS ÉLÉMENTS PARTAGÉS?}

Le PLU, par le PADD, est un projet qui doit garantir les enjeux techniques et socio-économiques indissociables d'un développement qui se traduisent par un ensemble d'aménagements évoluant selon des changements sociaux intrinsèques. L'intérêt de coordonner projet et territoire repose aussi sur l'ambition des acteurs à vouloir générer de nouvelles synergies, et tenter de fédérer un équilibre entre projets individuels et collectifs. « Le projet est destiné à mobiliser les énergies autour des enjeux, il acquiert simultanément une valeur symbolique pour les acteurs partie prenante et une valeur opératoire dans la mesure où il oriente une action concrète à promouvoir » (Boutinet, 1999). Toutefois, considérer l'ensemble des projets des acteurs d'un territoire nécessite un travail important de capitalisation des actions politiques et techniques, qui pour nous semble avoir été tenté par les élus de Billom dans le rapport et le zonage final du PLU.

Ces documents proposent une gestion des espaces agricoles répartis sur divers secteurs intégrant deux dynamiques de gestion : «état » et «projet ». Le tableau 2 représente les intentions liées à la gestion «état» (Deverre, 2005), une agriculture figée dans son état actuel, et une agriculture «projet» jouant sur sa multifonctionnalité. Ce tableau articule deux entrées : les trois types de zonages possibles dans un PLU (Agricole, Urbanisable, Naturel) et les deux types d'intentions qui leurs sont rattachées Nous avons choisi de représenter sur bloc-diagramme le paysage que le zonage du PLU retenu permettrait d'observer en 2020.

À partir de cette image, modélisant les éléments retenus dans le PLU, nous constatons que les espaces agricoles auront diminué par rapport à aujourd'hui. En effet, 15 ha vont être mobilisés pour les futures zones à urbaniser en périphérie du bourg existant. Cela montre clairement les intentions des élus de privilégier le projet d'accueil de nouvelles populations et d'un habitat individuel. L'enquête publique n’a pas révélée de désaccord des exploitants pour cette décision, ni pour le projet du carrefour giratoire, ou celui du chemin de randonnée. De plus, sur la future 


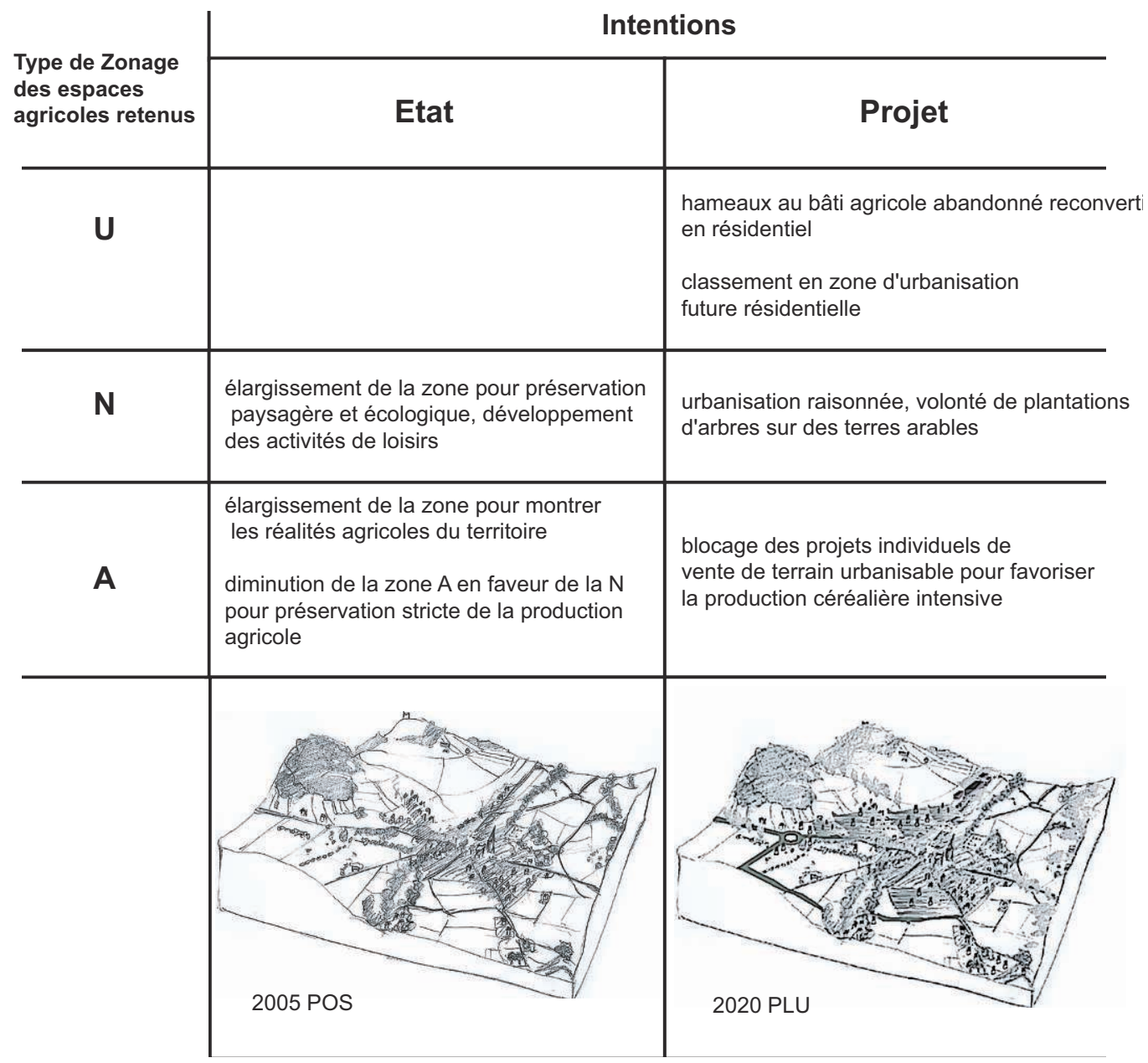

Tableau 2 : Évolution du POS en PLU

Comparison between the last and the new Urban Local Planning

zone à urbaniser, l'attente des exploitants de pouvoir conserver des parcelles non mitées est prise en compte : l'urbanisation au coup par coup est exclue.

En revanche, les projets particuliers des agriculteurs de diversification pour une multifonctionnalité des espaces agricoles n'apparaissent pas. Les zones A relèvent des intentions du groupe des agriculteurs actifs de moins de 50 ans et de la Chambre d'Agriculture de préserver une agriculture « moderne ", céréalière intensive, respectant les attentes régionales de l'agglomération Clermontoise. Les possibilités de voir des productions biologiques et des réseaux de vente directe émerger sur Billom est une attente sociale finalement oubliée du PLU.

Ainsi, le projet du PLU pour l'agriculture ne relève pas d'une gestion des espaces agricoles à partir des projets individuels des exploitants, mais selon une intention de préservation de ces espaces au nom de la nature, du paysage en accord avec un développement agricole économique, sans prendre plus en compte les enjeux écologiques et sociétaux de ce type d'agriculture. Finalement l'intention d'une agriculture « projet » sur un territoire périurbain n’apparaîtrait pas seulement par une mise en concurrence des zones A avec les zones U, mais aussi avec les zones $\mathrm{N}$. 


\section{L'AGRICULTURE ENTRE LES ZONES A ET N DU PLU}

Les élus et le bureau d'étude admettent au préalable leur méconnaissance des espaces agricoles, ce qui favorise leur volonté de participer à la démarche et de contribuer au débat. Leurs avis sur les valeurs paysagères sont partagés entre fonctions techniques et esthétiques. La carte du PLU devient pour eux, un compromis issu des différentes phases de débat. Néanmoins, nous constatons que les intentions politiques, au nom de "l'intérêt général et du cadre de vie », à l'échelle d'aménagement, ne permettent pas aux intentions individuelles de s'opposer. Pourtant, le graphique (figure 4) nous montre bien que ce sont les intentions à l'échelle de l'objet et de l'opération qui dominent dans les discours, c'est-à-dire les éléments que les acteurs connaissent, gèrent ou pratiquent individuellement. Certains exploitants agricoles mettent même en avant des intentions sur la production et la réserve foncière de l'espace agricole justifiant de nombreux investissements (drainage, irrigation, plantation de haies).

Ainsi, le choix de zoner en $\mathrm{A}$ ou en $\mathrm{N}$ a été conçu de manière à ne pas modifier le projet individuel des producteurs, mais celui de ceux qui veulent rendre l'espace constructible. Le zonage A va alors être pensé non pas seulement pour le maintien, et encore moins le développement de l'activité agricole, mais surtout pour le contrôle de l'étalement urbain. Le zonage en $\mathrm{N}$ des pentes des Turlurons ne va pas empêcher le mode de production intensive céréalière, mais faire valider par tous la valeur identitaire et paysagère du massif.

Le zonage en $\mathrm{N}$ avec des extensions d'urbanisation a été fortement débattu lors des ateliers participatifs du fait, d'une part, d'une incompréhension de la part de céréaliers de classer des parcelles en $\mathrm{N}$ au nom du paysage et parce qu'elles sont sur des pentes, alors que leurs pratiques culturales sont identiques à celles de la plaine. D'autre part, les architectes et les élus ont argumenté ce zonage par les valeurs d'un passé identitaire par des fonctions paysagères, environnementales et écologiques de l'espace agricole, sans réellement prendre en considération les types de production agricole qui la composent et qui pourtant leur avaient été présentés. Le rapport du PLU cite à ce propos : «Ont été intégrées à la zone $\mathrm{N}$ dans un souci de préservation des éléments paysagers structurants identifiés dans le cadre du PADD. Il ne s'agit en aucun cas de nier le caractère agricole de ses sites aujourd'hui exploités mais bien de faire en sorte que ces espaces constitutifs de l'identité de Billom soient préservés de toute forme d'urbanisation exception faites des quelques constructions déjà existantes. »

En lien avec cette remarque, environ 120 ha de zones A ont ainsi été converties en N, pour privilégier à la fois la production agricole dans un respect de la préservation environnementale et le développement raisonné des constructions. L'argument retenu dans le PLU est que « le zonage A n'étant adapté qu'aux constructions liées à une exploitation agricole, [...] les quelques parcelles bâties indépendantes ont été classées en zone $\mathrm{N}$ afin de permettre leur éventuel changement de destination et leur extension modérée ».

Le zonage N a ainsi pour « projet » d'éluder l'agriculture mais d'accepter une extension « modérée » des constructions existantes, en mobilisant l'argument, d'un zonage « état » au nom de l'environnement et du paysage. Cette zone $\mathrm{N}$ deviendrait-elle un espace «fourre-tout », permettant du coup de concilier, plutôt que de partager, toutes les intentions?

\section{PLU ET MÉTHODE PARTICIPATIVE : QUEL BILAN?}

L'IMVP met en avant l'intérêt de l'approche par la négative : citer ce qu'on ne veut pas voir pour mieux décider ce que l'on souhaite. Cette approche a présenté plusieurs avantages pour les acteurs. Lors de l'atelier participatif, les acteurs ont pu confronter leur opinion sur ce qui plaît ou déplaît, parler des lieux connus ou méconnus, et surtout tenter de définir les éléments qui sont génériques ou spécifiques au territoire. Nous avons également constaté que l'approche négative a permis de diminuer les tensions et de voir comment les intentions individuelles (révélées par les entretiens) s'associent ou s'opposent lors du débat. Pour un exploitant, la négation a semblé plus évidente pour comprendre au regard d'un autre l'intérêt de ces pratiques : « tu ne veux pas ça, et 
pourquoi?... mais c'est chez moi ça, bon alors il faut trouver une autre solution ou alors si c'est chez moi ça ne changera pas!».

L'usage des représentations graphiques, telles que le bloc-diagramme, associé à la négation, facilite la localisation des objets paysagers génériques qu'on ne souhaite pas (i.e., l'espace pavillonnaire figure 2a). Nous constatons cependant que tous les éléments débattus ne sont pas retenus dans le PLU, peut-être du fait d'une concertation réduite aux agriculteurs. D'autres éléments, importants pour d'autres acteurs absents, seront oubliés ou méconnus des participants.

Mobiliser les objets paysagers pour la compréhension des intentions sur l'espace peut s'inscrire dans l'analyse des postures personnelles et professionnelles qui influence l'acteur dans son discours et ses représentations. En effet, un acteur est « un individu qui est simultanément habitant, citoyen, parent d'élève, employé » (Joliveau, 2001). Nous avons constaté que l'acteur peut parler des éléments paysagers qu'il connaît mais aussi qu'il imagine. Ainsi un objet paysager permet de fédérer des opinions selon des approches et des échelles d'appréciation, de perçu et de vécu de l'espace différencié. L'ensemble de ces échelles, dans un espace périurbain, est perçu par divers acteurs indépendamment de leur réalité, balancé entre des logiques publiques et privées (Donadieu, 2000; Tizon, 2006), mais aussi endogènes et exogènes au territoire. Ce qui porte l'intérêt d'utiliser des objets communément connus, mais qui, du fait de leur matérialité par leur généricité et leur ancrage spatial, peuvent afficher une certaine neutralité.

Un autre intérêt, révélé par notre approche, est qu'un objet observé à son échelle peut-être très déprécié, mais pensé à l'échelle d'aménagement, il devient un composant de qualité, dans un panorama par exemple. Ainsi son intégration à des échelles différente va lui permettre de conférer des acceptations ou des actions d'aménagements différenciés. Il est alors intéressant de voir en quoi l'articulation des échelles d'observation avec la dimension symbolique des objets paysagers permet de construire une image qui va au-delà de l'intention.

\section{Conclusion}

La plupart des projets de planification sont traduits par une cartographie de zonages davantage déterminés politiquement que techniquement, puisqu'il «n'existe pas d'aménagement neutre de l'espace »(Gumuchian, 1991). Notre démarche argumente cette remarque notamment par rapport aux enjeux de l'appropriation spatiale selon les échelles de représentations et d'intentions sur ces espaces. L'articulation des représentations spatiales et sociales peut effectivement permettre de reconnaître collectivement des intentions individuelles pouvant être récupérées par le collectif. Alors que l'intérêt général, à l'échelle d'aménagement, a tendance à diluer l'intérêt privatif.

Il est intéressant de remarquer que dans leur quotidien, les individus n'éprouvent pas la nécessité de mettre en avant leur rapport à l'espace. En revanche, la mise en réflexion d'un projet, d'une planification devient un déclencheur des dispositifs spatiaux pratiqués et représentés afin que l'individu ou le collectif positionne ses intentions face à une opportunité d'aménagement (Tizon, 2006). Ainsi notre démarche sert d'outil d'information et de partage de connaissances entre agriculteurs et autres acteurs. Les supports (bloc-diagramme, cartographie...) sont facilement lisibles pour les participants et appropriables par les responsables du projet comme outil de communication et de sensibilisation auprès d'autres partenaires à différentes échelles (habitants, services de l'État...). Les démarches participatives constituent des événements phares pour l'implication d'acteurs rarement sollicités dans l'aide à la décision d'un zonage. Les élus et l'urbaniste ont apprécié l'objectif d'opérationnalité de notre démarche. Nous reconnaissons également l'importance de notre action répartie sur la durée du PLU, en amont et en aval, pour laisser justement le temps aux acteurs d'appréhender l'importance de leur projet en dedans et en dehors de la procédure.

Notre expérimentation valide l'intérêt de développer de nouvelles méthodes à la mise en œuvre d'un PLU pour une meilleure compréhension des territoires communaux, voire intercommunaux, et de leur contexte agricole. La connaissance partagée, en termes de valeurs et de techniques permet de révéler, en aval et au fur et à mesure de la procédure, des intentions de gestion pou- 
vant s'adapter aux changements rapides caractéristiques des territoires périurbains à une échelle locale et plus globale. Si l'intérêt du PLU repose bien sur sa faculté d'être prospectif en plus d'être régulateur, la pluralité des modalités de concertation pour partager ces connaissances est encore à développer.

\section{Bibliographie}

Boutinet (J.-P.), 1990. - Anthropologie du projet, Paris, PUF, 350 p.

Code de l'Urbanisme, [www.legifrance.gouv.fr].

Deverre (C.), 2005. - « Les dispositifs réglementaires et institutionnels pour la gestion des usages agricoles du territoire », dans Launent (C.), Thinon (P.) (dir.), Agriculture et territoires, Paris, Hermès, p. 269281.

DonAdieu (P.), 2000. - « Les processus paysagers comme indicateurs de la construction des paysages périurbains ", Physionomies, dynamiques et fonctionnements des paysages périurbains, Journées d'études des 3 et 4 octobre 2000, Université d'Avignon, p. 123-130.

FLeury (A.), 2005. - « L'agriculture dans la planification de l'Île-de-France : du vide urbain à la multifonctionnalité territoriale », Les Cahiers de la Multifonctionnalité, L'agriculture périurbaine, nº 8, Paris, INRA, p. 33-46.

Gumuchian (H.), 1991. - Représentation et Aménagement du territoire, Paris, Édition Anthropos Économica, $143 \mathrm{p}$.

JeAn (B.), Dionne (S.), 2007. - « la ruralité entre les appréciations statistiques et les représentations sociales : comprendre la reconfiguration socio-spatiale des territoires ruraux québécois », Recomposition des espaces Ruraux, Norois, n² 202-2007/1, Rennes, PUR, p. 9-19.

JoliveAu (T), 2001. - « La participation à la décision territoriale : dimension socio-géographique et enjeux informationnels d'une question politique », Geocarrefour, vol. 76, p. 25-45

Lardon (S.), Maurel (P.), Piveteau (V.), 2001. - Représentations spatiales et développement territorial, Paris, Hermès, $437 \mathrm{p}$.

Michelin (Y.), 2000. - « Le bloc-diagramme : une clé de compréhension des représentations du paysage chez les agriculteurs? », Cybergeo, $\mathrm{n}^{\circ} 118,11$ p., [www.cybergeo.eu/index1992.html]

Planchat (C.), Loudiy (S.), (sous-presse). - « Objets et représentations paysagères des territoires en périphérie d'agglomération, Illustration à partir du Pays de Billom Saint-Dier. (Puy-de-Dôme) », Vers les paysages de demain : outils iconographiques et ressources territoriales. Des représentations à la valorisation, $2^{\mathrm{e}}$ journées d'études du programme ACI, ENITA de Clermont-Ferrand.

SYCOMORE, 2007. - Plan Local d'Urbanisme de la commune de Billom, Rapport de présentation, FREYDFONT (J.-M.), Groupe SYCOMORE, Mairie de Billom (dir.), 101 p.

Tizon (P.), 2006. - «Qu'est-ce que le territoire? », dans Di MEo (G.) (dir.), Les territoires du quotidien, Paris, L'Harmattan, p. 17-34.

VAnier (M.), 2003. - « Le périurbain à l'heure du crapaud buffle : tiers espace de la nature, nature du tiers espace ", Revue de Géographie Alpine, tome 91, p. 79-89.

Cet article a été reçu le 27 avril 2008 et définitivement accepté le 20 janvier 2009. 